\title{
Asymptotic and measured large frequency separations ${ }^{\star}$
}

\author{
B. Mosser ${ }^{1}$, E. Michel ${ }^{1}$, K. Belkacem ${ }^{1}$, M. J. Goupil ${ }^{1}$, A. Baglin ${ }^{1}$, C. Barban ${ }^{1}$, J. Provost ${ }^{2}$, R. Samadi ${ }^{1}$, \\ M. Auvergne ${ }^{1}$, and C. Catala ${ }^{1}$ \\ ${ }^{1}$ LESIA, CNRS, Université Pierre et Marie Curie, Université Denis Diderot, Observatoire de Paris, 92195 Meudon Cedex, France \\ e-mail: benoit.mosser@obspm.fr \\ ${ }^{2}$ Université de Nice-Sophia Antipolis, CNRS UMR 7293, Observatoire de la Côte d'Azur, Laboratoire J.L. Lagrange, BP 4229 , \\ 06304 Nice Cedex 04, France
}

Received 25 September 2012 / Accepted 9 December 2012

\begin{abstract}
Context. With the space-borne missions CoRoT and Kepler, a large amount of asteroseismic data is now available and has led to a variety of work. So-called global oscillation parameters are inferred to characterize the large sets of stars, perform ensemble asteroseismology, and derive scaling relations. The mean large separation is such a key parameter, easily deduced from the radial-frequency differences in the observed oscillation spectrum and closely related to the mean stellar density. It is therefore crucial to measure it with the highest accuracy in order to obtain the most precise asteroseismic indices.

Aims. As the conditions of measurement of the large separation do not coincide with its theoretical definition, we revisit the asymptotic expressions used for analyzing the observed oscillation spectra. Then, we examine the consequence of the difference between the observed and asymptotic values of the mean large separation.

Methods. The analysis is focused on radial modes. We use series of radial-mode frequencies in published analyses of stars with solarlike oscillations to compare the asymptotic and observational values of the large separation. This comparison relies on the proper use of the second-order asymptotic expansion.

Results. We propose a simple formulation to correct the observed value of the large separation and then derive its asymptotic counterpart. The measurement of the curvature of the radial ridges in the échelle diagram provides the correcting factor. We prove that, apart from glitches due to stellar structure discontinuities, the asymptotic expansion is valid from main-sequence stars to red giants. Our model shows that the asymptotic offset is close to $1 / 4$, as in the theoretical development, for low-mass, main-sequence stars, subgiants and red giants.

Conclusions. High-quality solar-like oscillation spectra derived from precise photometric measurements are definitely better described with the second-order asymptotic expansion. The second-order term is responsible for the curvature observed in the échelle diagrams used for analyzing the oscillation spectra, and this curvature is responsible for the difference between the observed and asymptotic values of the large separation. Taking it into account yields a revision of the scaling relations, which provides more accurate asteroseismic estimates of the stellar mass and radius. After correction of the bias (6\% for the stellar radius and 3\% for the mass), the performance of the calibrated relation is about $4 \%$ and $8 \%$ for estimating, respectively, the stellar radius and the stellar mass for masses less than $1.3 M_{\odot}$; the accuracy is twice as bad for higher mass stars and red giants.
\end{abstract}

Key words. stars: oscillations - stars: interiors - methods: analytical - methods: data analysis

\section{Introduction}

The amount of asteroseismic data provided by the space-borne missions CoRoT and Kepler has given rise to ensemble asteroseismology. With hundreds of stars observed from the main sequence to the red giant branch, it is possible to study evolutionary sequences and to derive seismic indices from global seismic observational parameters. These global parameters can be measured prior to any complete determination of the individual mode frequencies and are able to provide global information on the oscillation spectra (e.g., Michel et al. 2008). For instance, the mean large separation $\Delta v$ between consecutive radial-mode frequencies and the frequency $v_{\max }$ of maximum oscillating signal are widely used to provide estimates of the stellar mass and radius (e.g., Kallinger et al. 2010; Mosser et al. 2010). Almost all other scaling relations make use of $\Delta v$, as, for instance, the scaling reations governing the amplitude of the oscillation signal

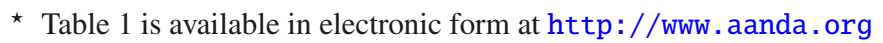

(e.g., Stello et al. 2011; Mosser et al. 2012a). In case an oscillation spectrum is determined with a low signal-to-noise ratio, the mean large separation is the single parameter that can be precisely measured (e.g., Bedding et al. 2001; Mosser et al. 2009; Gaulme et al. 2010).

The definition of the large separation relies on the asymptotic theory, valid for large values of the eigenfrequencies, corresponding to large values of the radial order. However, its measurement is derived from the largest peaks seen in the oscillation spectrum in the frequency range surrounding $v_{\max }$, thus in conditions that do not directly correspond to the asymptotic relation. This difference can be taken into account in comparisons with models for a specific star, but not in the consideration of large sets of stars for statistical studies.

In asteroseismology, ground-based observations with a limited frequency resolution have led to the use of simplified and incomplete forms of the asymptotic expansion. With CoRoT and Kepler data, these simplified forms are still in use, but observed uncertainties on frequencies are much reduced. As for the 
Sun, one may question using the asymptotic expansion, since the quality of the data usually allows one to go beyond this approximate relation. Moreover, modeling has shown that acoustic glitches due to discontinuities or important gradients in the stellar structure may hamper the use of the asymptotic expansion (e.g., Audard \& Provost 1994).

In this work, we aim to use the asymptotic relation in a proper way to derive the generic properties of a solar-like oscillation spectrum. It seems therefore necessary to revisit the different forms of asymptotic expansion since $\Delta v$ is introduced by the asymptotic relation. We first show that it is necessary to use the asymptotic expansion including its second-order term, without simplification compared to the theoretical expansion. Then, we investigate the consequence of measuring the large separation at $v_{\max }$ and not in asymptotic conditions. The relation between the two values, $\Delta v_{\mathrm{as}}$ for the asymptotic value and $\Delta v_{\mathrm{obs}}$ for the observed one, is developed in Sect. 2. The analysis based on radial-mode frequencies found in the literature is carried out in Sect. 3 to quantify the relation between the observed and asymptotic parameters, under the assumption that the secondorder asymptotic expansion is valid for describing the radial oscillation spectra. We verify that this hypothesis is valid when we consider two regimes, depending on the stellar evolutionary status. We discuss in Sect. 4 the consequences of the relation between the observed and asymptotic parameters. We also use the comparison of the seismic and modeled values of the stellar mass and radius to revise the scaling relations providing $M$ and $R$ estimates.

\section{Asymptotic relation versus observed parameters}

\subsection{The original asymptotic expression}

The oscillation pattern of low-degree oscillation pressure modes can be described by a second-order relation (Eqs. (65)-(74) of Tassoul 1980). This approximate relation is called asymptotic, since its derivation is strictly valid only for large radial orders. The development of the eigenfrequency $v_{n, \ell}$ proposed by Tassoul includes a second-order term, namely, a contribution in $1 / v_{n, \ell}$ :

$v_{n, \ell}=\left(n+\frac{\ell}{2}+\varepsilon\right) \Delta v-\left[\ell(\ell+1) d_{0}+d_{1}\right] \frac{\Delta v^{2}}{v_{n, \ell}}$

where $n$ is the p-mode radial order, $\ell$ is the angular degree, $\Delta v$ is the large separation, and $\varepsilon$ is a constant term. The terms $\Delta v$ and $\varepsilon$ are discussed later; the dimensionless term $d_{0}$ is related to the gradient of sound speed integrated over the stellar interior; $d_{1}$ has a complex form.

\subsection{The asymptotic expansion used in practice}

When reading the abundant literature on asteroseismic observations, the most common forms of the asymptotic expansion used for interpreting observed low-degree oscillation spectra (e.g., Mosser et al. 1991; Bedding et al. 2001; Bouchy et al. 2005, for ground-based observations) are similar to the approximate form

$v_{n, \ell} \simeq\left(n+\frac{\ell}{2}+\varepsilon_{0}\right) \Delta v_{0}-\ell(\ell+1) D_{0}$.

Compared to Eq. (1), the contribution of $d_{1}$ and the variation of the denominator varying as $v_{n, \ell}$ are both omitted. This omission derives from the fact that ground-based observations have a too

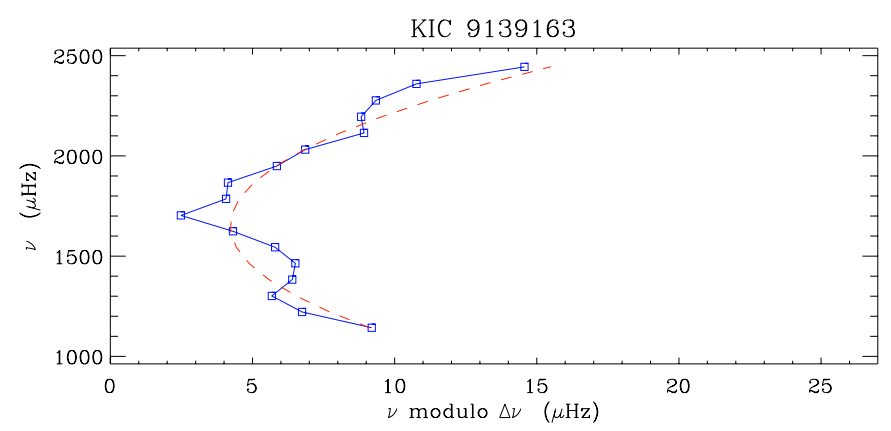

Fig. 1. Échelle diagram of the radial modes of the star KIC 9139163 (from Appourchaux et al. 2012). The red dashed line indicates the quadratic fit that mimics the curvature.

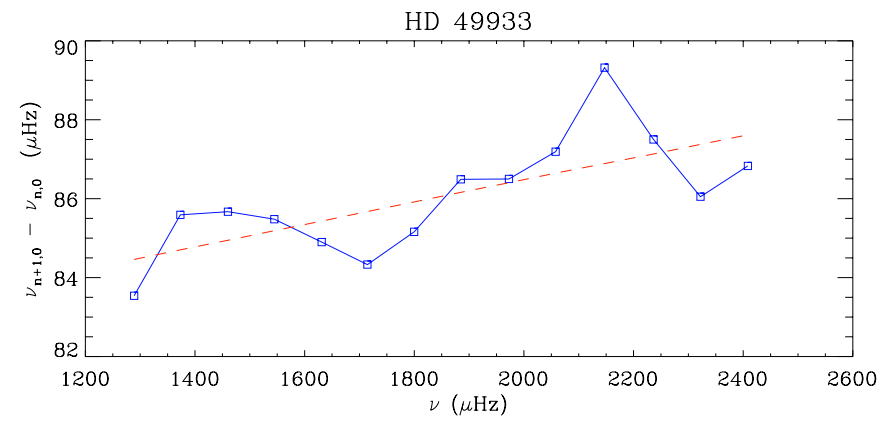

Fig. 2. Variation of the large separations $v_{n+1,0}-v_{n, 0}$ as a function of $v_{n, 0}$ for the star HD 49933 (from Benomar et al. 2009). The red dashed line indicates a linear fit.

coarse frequency resolution. Equation (2) is still in use for spaceborne observations that provide a much better frequency resolution (e.g., Campante et al. 2011; White et al. 2012; Corsaro et al. 2012). The large separation $\Delta v_{0}$ and the offset $\varepsilon_{0}$ are supposed to play the roles of $\Delta v$ and $\varepsilon$ in Eq. (1): this is not strictly exact, as shown later.

Observationally, the terms $\Delta v_{0}$ and $D_{0}$ can be derived from the mean frequency differences

$\Delta v_{0}=\left\langle v_{n+1, \ell}-v_{n, \ell}\right\rangle$,

$D_{0}=\left\langle\frac{1}{4 \ell+6}\left(v_{n, \ell}-v_{n-1, \ell+2}\right)\right\rangle$,

where the square brackets represent the mean values in the observed frequency range. For radial modes, the asymptotic expansion reduces to

$v_{n, 0}=\left(n+\varepsilon_{0}\right) \Delta v_{0}$.

Clearly, this form cannot account for an accurate description of the radial-mode pattern, since the échelle diagram representation shows an noticeable curvature for most stars with solar-like oscillations at all evolutionary stages. This curvature, always with the same concavity sign, is particularly visible in red giant oscillations (e.g., Mosser et al. 2011; Kallinger et al. 2012), which show solar-like oscillations at low radial order (e.g., De Ridder et al. 2009; Bedding et al. 2010a). Figure 1 shows a typical example of the non-negligible curvature in the échelle diagram of a main-sequence star with many radial orders. The concavity corresponds equivalently to a positive gradient of the large separation (Fig. 2). This gradient is not reproduced by Eq. (5), stressing that this form of the asymptotic expansion is not adequate for reporting the global properties of the radial modes observed with enough precision. Hence, it is necessary to revisit the use of the 
asymptotic expression for interpreting observations and to better account for the second-order term.

\subsection{Radial modes depicted by second-order asymptotic expansion}

We have chosen to restrict the analysis to radial modes. We express the second-order term varying in $v^{-1}$ of Eq. (1) with a contribution in $n^{-1}$ :

$v_{n, 0}=\left(n+\varepsilon_{\mathrm{as}}+\frac{A_{\mathrm{as}}}{n}\right) \Delta v_{\mathrm{as}}$.

Compared to Eq. (1), we added the subscript as to the different terms in order to make clear that, contrary to Eq. (2), we respect the asymptotic condition. We replaced the second-order term in $1 / v$ by a term in $1 / n$ instead of $1 /\left(n+\varepsilon_{\text {as }}\right)$, since the contribution of $\varepsilon_{\mathrm{as}}$ in the denominator can be considered as a third-order term in $n$.

The large separation $\Delta v_{\mathrm{as}}$ is related to the stellar acoustic diameter by

$\Delta v_{\mathrm{as}}=\left(2 \int_{0}^{R} \frac{\mathrm{d} r}{c}\right)^{-1}$,

where $c$ is the sound speed. We note that $\Delta v_{0}$, different from the asymptotic value $\Delta v_{\text {as }}$, cannot directly provide the integral value of $1 / c$. We also note that the offset $\varepsilon_{\text {as }}$ has a fixed value in the original work of Tassoul:

$\varepsilon_{\mathrm{as}, \text { Tassoul }}=\frac{1}{4}$.

In the literature, one also finds that $\varepsilon_{\text {as }}=1 / 4+a(v)$, where $a(v)$ is determined by the properties of the near-surface region (Christensen-Dalsgaard \& Perez Hernandez 1992).

The second-order expansion is valid for large radial orders only, when the second-order term $A_{\text {as }} / n$ is small. This means that the large separation $\Delta v_{\text {as }}$ corresponds to the frequency difference between radial modes at high frequency only, but not at $v_{\max }$. We note that the second-order term can account for the curvature of the radial ridge in the échelle diagram, with a positive value of $A_{\text {as }}$ for reproducing the sign of the observed concavity.

\subsection{Taking into account the curvature}

In practice, the large separation is necessarily obtained from the radial modes with the largest amplitudes observed in the oscillation pattern around $v_{\max }$ (e.g., Mosser \& Appourchaux 2009). In order to reconcile observations at $v_{\max }$ and asymptotic expansion at large frequency, we must first consider the curvature of the ridge. To enhance the quality of the fit of radial modes in red giants, Mosser et al. (2011) have proposed including the curvature of the radial ridge with the expression

$v_{n, 0}=\left(n+\varepsilon_{\mathrm{obs}}+\frac{\alpha_{\mathrm{obs}}}{2}\left[n-n_{\mathrm{max}}\right]^{2}\right) \Delta v_{\mathrm{obs}}$,

where $\Delta v_{\mathrm{obs}}$ is the observed large separation, measured in a wide frequency range around the frequency $v_{\max }$ of maximum oscillation amplitude, $\alpha_{\mathrm{obs}}$ is the curvature term, and $\varepsilon_{\mathrm{obs}}$ is the offset. We have also introduced the dimensionless value of $v_{\max }$, defined by $n_{\max }=v_{\max } / \Delta v_{\text {obs }}$. Similar fits have already been proposed for the oscillation spectra of the Sun (Christensen-Dalsgaard \& Frandsen 1983; Grec et al. 1983; Scherrer et al. 1983), of $\alpha$ Cen A (Bedding et al. 2004), $\alpha$ Cen B (Kjeldsen et al. 2005), HD 203608 (Mosser et al. 2008b), Procyon (Mosser et al. 2008a), and HD 46375 (Gaulme et al. 2010). In fact, such a fit mimics a second-order term and provides a linear gradient in large separation:

$\frac{v_{n+1,0}-v_{n-1,0}}{2}=\left(1+\alpha_{\mathrm{obs}}\left[n-n_{\max }\right]\right) \Delta v_{\mathrm{obs}}$.

The introduction of the curvature may be considered as an empirical form of the asymptotic relation. It reproduces the second-order term of the asymptotic expansion, which has been neglected in Eq. (2), with an unequivocal correspondence. It relies on a global description of the oscillation spectrum, which considers that the mean values of the seismic parameters are determined in a large frequency range around $v_{\max }$ (Mosser \& Appourchaux 2009). Such a description has shown interesting properties when compared to a local one that provides the large separation from a limited frequency range only around $v_{\max }$ (Verner et al. 2011; Hekker et al. 2012).

It is straightforward to make the link between both asymptotic and observed descriptions of the radial oscillation pattern with a second-order development in $\left(n-n_{\max }\right) / n_{\max }$ of the asymptotic expression. From the identification of the different orders in Eqs. (6) and (9) (constant, varying in $n$ and in $n^{2}$ ), we then get

$\Delta v_{\mathrm{as}}=\Delta v_{\mathrm{obs}}\left(1+\frac{n_{\mathrm{max}} \alpha_{\mathrm{obs}}}{2}\right)$

$A_{\mathrm{as}}=\frac{\alpha_{\mathrm{obs}}}{2} \frac{n_{\mathrm{max}}^{3}}{1+n_{\max } \frac{\alpha_{\mathrm{obs}}}{2}}$,

$\varepsilon_{\mathrm{as}}=\frac{\varepsilon_{\mathrm{obs}}-n_{\mathrm{max}}^{2} \alpha_{\mathrm{obs}}}{1+n_{\max } \frac{\alpha_{\mathrm{obs}}}{2}}$.

When considering that the ridge curvature is small enough $\left(n_{\max } \alpha_{\text {obs }} / 2 \ll 1\right), A_{\text {as }}$ and $\varepsilon_{\text {as }}$ become

$A_{\mathrm{as}} \simeq \frac{\alpha_{\mathrm{obs}}}{2} n_{\mathrm{max}}^{3}$

$\varepsilon_{\mathrm{as}} \simeq \varepsilon_{\mathrm{obs}}\left(1-\frac{n_{\mathrm{max}} \alpha_{\mathrm{obs}}}{2}\right)-n_{\mathrm{max}}^{2} \alpha_{\mathrm{obs}}$.

These developments provide a reasonable agreement with the previous exact correspondence between the asymptotic and observed forms.

The difference between the observed and asymptotic values of $\varepsilon$ includes a systematic offset in addition to the rescaling term $\left(1-n_{\max } \alpha_{\mathrm{obs}} / 2\right)$. This comes from the fact that the measurement of $\varepsilon_{\mathrm{obs}}$ significantly depends on the measurement of $\Delta v_{\mathrm{obs}}$ : a relative change $\eta$ in the measurement of the large separation translates into an absolute change of the order of $-n_{\max } \eta$. This indicates that the measurement of $\varepsilon_{\mathrm{obs}}$ is difficult since it includes large uncertainties related to all effects that affect the measurement of the large separation, such as structure discontinuities or significant gradients of composition (Miglio et al. 2010; Mazumdar et al. 2012).

\section{5. Échelle diagrams}

The échelle diagrams in Fig. 3 compare the radial oscillation patterns folded with $\Delta v_{\mathrm{obs}}$ or $\Delta v_{\mathrm{as}}$. In practice, the folding is naturally based on the observations of quasi-vertical ridges and provides $\Delta v_{\mathrm{obs}}$ observed at $v_{\max }$ (Fig. 3, top). A folding based on 

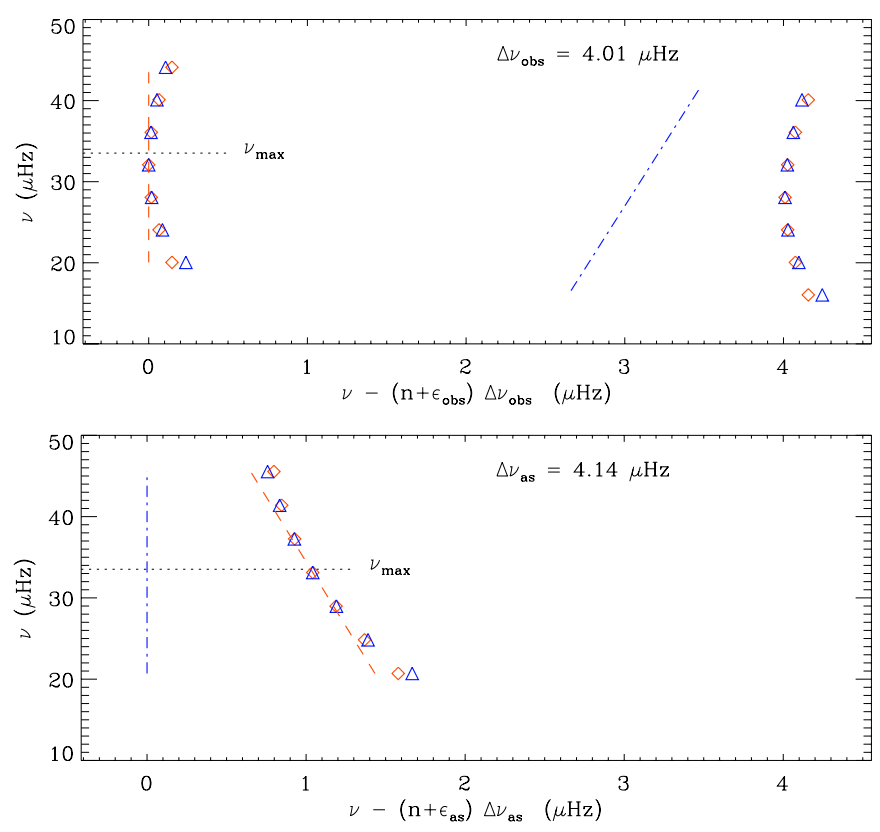

Fig. 3. Échelle diagrams of the radial modes of a typical red-clump giant, comparing the asymptotic expansion (Eq. (6), blue triangles) and the development describing the curvature (Eq. (9), red diamonds). Top: diagram based on $\Delta v_{\text {obs }}$ observed at $v_{\max }$; the dashed line indicates the vertical asymptotic line at $v_{\max }$; the dot-dashed line indicates the asymptotic line at high frequency. For clarity, the ridge has been duplicated modulo $\Delta v_{\mathrm{obs}}$. Bottom: diagram based on $\Delta v_{\mathrm{as}}$; the dot-dashed line indicates the vertical asymptotic line at high frequency.

$\Delta v_{\text {as }}$ would not show any vertical ridge in the observed domain (Fig. 3, bottom). In Fig. 3 we have also compared the asymptotic spectrum based on the parameters $\Delta v_{\mathrm{as}}, A_{\mathrm{as}}$, and $\varepsilon_{\mathrm{as}}$ to the observed spectrum, obeying Eq. (9). Perfect agreement is naturally met for $v \simeq v_{\max }$. Differences vary as $\alpha_{\text {obs }}\left(n-n_{\max }\right)^{3}$. They remain limited to a small fraction of the large separation, even for the orders far from $n_{\max }$, so that the agreement of the simplified expression is satisfactory in the frequency range where modes have appreciable amplitudes. Comparison of the échelle diagrams illustrates that the observable $\Delta v_{\mathrm{obs}}$ significantly differs from the physically-grounded asymptotic value $\Delta v_{\mathrm{as}}$.

\section{Data and analysis}

\subsection{Observations: main-sequence stars and subgiants}

Published data allowed us to construct a table of observed values of $\alpha_{\mathrm{obs}}$ and $\varepsilon_{\mathrm{obs}}$ as a function of the observed large separation $\Delta v_{\text {obs }}$ (Table 1, with 100 stars). We considered observations of subgiants and main-sequence stars observed by CoRoT or by Kepler. We also made use of ground-based observations and solar data. All references are given in the caption of Table 1.

We have considered the radial eigenfrequencies, calculated local large spacings $v_{n+1,0}-v_{n, 0}$, and derived $\Delta v_{\text {obs }}$ and $\alpha_{\text {obs }}$ from a linear fit corresponding to the linear gradient given by Eq. (10). The term $\varepsilon_{\text {obs }}$ is then derived from Eq. (9). With the help of Eqs. (11)-(13), we analyzed the differences between the observables $\Delta v_{\mathrm{obs}}, \varepsilon_{\mathrm{obs}}, \alpha_{\mathrm{obs}}$ and their asymptotic counterparts $\Delta v_{\mathrm{as}}$, $\varepsilon_{\mathrm{as}}$, and $A_{\mathrm{as}}$. We have chosen to express the variation with the parameter $n_{\max }$, rather than $\Delta v_{\mathrm{obs}}$ or $v_{\max }$. Subgiants have typically $n_{\max } \geq 15$, and main-sequence stars $n_{\max } \geq 18$.

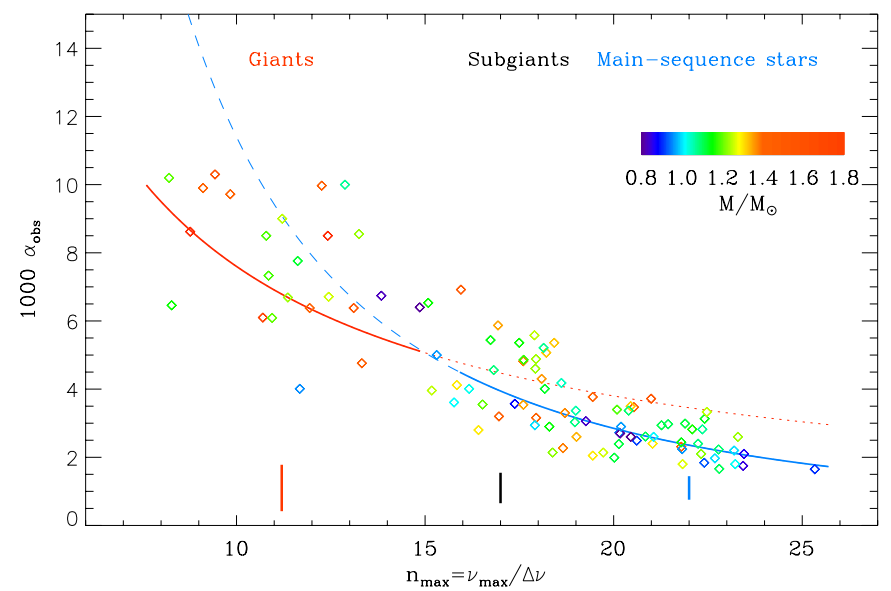

Fig. 4. Curvature $10^{3} \alpha_{\text {obs }}$ as a function of $n_{\max }=v_{\max } / \Delta v$. The thick line corresponds to the fit in $n_{\max }^{-1}$ established for red giants, and the dotted line to its extrapolation towards larger $n_{\max }$. The dashed line provides an acceptable fit in $n_{\max }^{-2}$ valid for main-sequence stars. Error bars indicate the typical $1-\sigma$ uncertainties in three different domains. The color code of the symbols provides an estimate of the stellar mass; the colors of the lines correspond to the different regimes.

\subsection{Observations: red giants}

We also considered observations of stars on the red giant branch that have been modelled. They were analyzed exactly as the lessevolved stars. However, this limited set of stars cannot represent the diversity of the thousands of red giants already analyzed in both the CoRoT and Kepler fields (e.g., Hekker et al. 2009; Bedding et al. 2010a; Mosser et al. 2010; Stello et al. 2010). We note, for instance, that their masses are higher than the mean value of the largest sets. Furthermore, because the number of excited radial modes is much more limited than for main-sequence stars (Mosser et al. 2010), the observed seismic parameters suffer from a large spread. Therefore, we also made use of the mean relation found for the curvature of the red giant radial oscillation pattern (Mosser et al. 2012b)

$\alpha_{\mathrm{obs}, \mathrm{RG}}=0.015 \Delta v_{\mathrm{obs}}^{-0.32}$.

This relation, when expressed as a function of $n_{\max }$ and taking into account the scaling relation $\Delta v \propto v_{\max }^{0.75}$ (e.g., Hekker et al. 2011), gives $\alpha_{\mathrm{obs}, \mathrm{RG}}=0.09 n_{\max }^{-0.96}$. The exponent of this scaling relation is close to -1 , so that for the following study we simply consider the fit

$\alpha_{\mathrm{obs}, \mathrm{RG}}=2 a_{\mathrm{RG}} n_{\mathrm{max}}^{-1}$,

with $a_{\mathrm{RG}}=0.038 \pm 0.002$. Red giants have typically $n_{\max } \leq 15$.

\subsection{Second-order term $A_{\text {as }}$ and curvature $\alpha_{\text {obs }}$}

We first examined the curvature $\alpha_{\text {obs }}$ as a function of $n_{\max }$ (Fig. 4), since this term governs the relation between the asymptotic and observed values of the large separation (Eq. (11)). A large spread is observed because of the acoustic glitches caused by structure discontinuities (e.g., Miglio et al. 2010). Typical uncertainties of $\alpha_{\text {obs }}$ are about $20 \%$. We are interested in the mean variation of the observed and asymptotic parameters, so that the glitches are first neglected and later considered in Sect. 4.1. The large spread of the data implies that, as is well known, the 


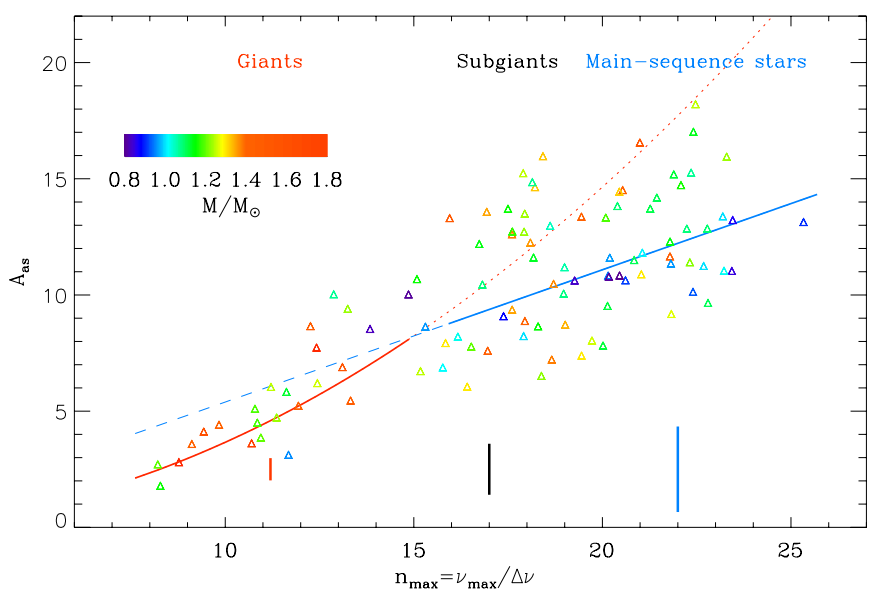

Fig. 5. Same as Fig. 4, for the second-order asymptotic term $A_{\text {as }}$ as a function of $n_{\max }$. The fits of $A_{\text {as }}$ in the different regimes are derived from the fits of $\alpha_{\mathrm{obs}}$ in Fig. 4 and the relation provided by Eq. (12).

asymptotic expansion cannot precisely relate all of the features of a solar-like oscillation spectrum. However, this spread does not invalidate the analysis of the mean evolution of the observed and asymptotic parameters with frequency.

The fit of $\alpha_{\text {obs }}$ derived from red giants, valid when $n_{\max }$ is in the range [7, 15], does not hold for less-evolved stars with larger $n_{\max }$ (Fig. 4). It could reproduce part of the observed curvature of subgiants but yields too large values in the mainsequence domain. In order to fit main-sequence stars and subgiants, it seems necessary to modify the exponent of the relation $\alpha_{\text {obs }}\left(n_{\max }\right)$. When restricted to main-sequence stars, the fit of $\alpha_{\text {obs }}\left(n_{\max }\right)$ provides an exponent of about $-2 \pm 0.3$. We thus chose to fix the exponent to the integer value -2 :

$\alpha_{\mathrm{obs}, \mathrm{MS}}=2 a_{\mathrm{MS}} n_{\mathrm{max}}^{-2}$,

with $a_{\mathrm{MS}}=0.57 \pm 0.02$. Having a different fit compared to the red giant case (Eq. (16)) is justified in Sect. 3.5: even if a global fit in $n_{\max }^{-1.5}$ should reconcile the two regimes, we keep the two regimes since we also have to consider the fits of the other asymptotic parameters, especially $\varepsilon_{\mathrm{obs}}$. We also note a gradient in mass: low-mass stars have systematically lower $\alpha_{\text {obs }}$ than high-mass stars. Masses were derived from the seismic estimates when modeled masses are not available (Table 1). At this stage, it is however impossible to take this mass dependence into consideration.

As a consequence of Eq. (14), we find that the second-order asymptotic term $A_{\text {as }}$ scales as $n_{\max }^{2}$ for red giants and as $n_{\max }$ for less-evolved stars (Fig. 5). We note, again, a large spread of the values, which is related to the acoustic glitches.

\subsection{Large separations $\Delta v_{\mathrm{as}}$ and $\Delta v_{\mathrm{obs}}$}

The asymptotic and observed values of the large separations of the set of stars are clearly distinct (Fig. 6). According to Eq. (11), the correction from $\Delta v_{\mathrm{obs}}$ to $\Delta v_{\mathrm{as}}$ has the same relative uncertainty as $\alpha_{\text {obs }}$. The relative difference between $\Delta v_{\text {as }}$ and $\Delta v_{\text {obs }}$ increases when $n_{\max }$ decreases and reaches a constant maximum value of about $4 \%$ in the red giant regime, in agreement with Eq. (17). Their difference is reduced at high frequency for subgiants and main-sequence stars, as in the solar case, where high radial orders are observed, but still of the order to $2 \%$. This

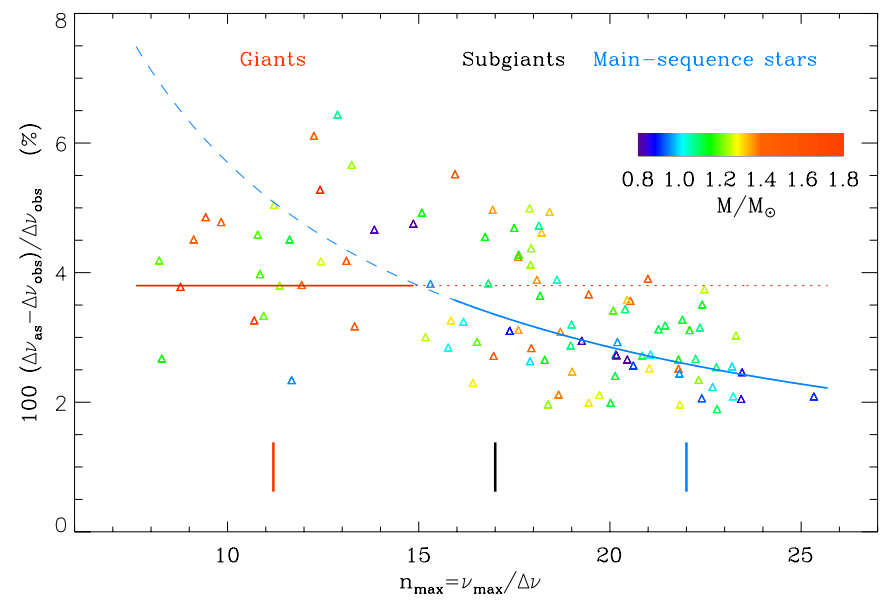

Fig. 6. Same as Fig. 4, for the relative difference of the large separations, equivalent to $n_{\max } \alpha_{\mathrm{obs}} / 2$, as a function of $n_{\max }$.

relative difference, even if small, depends on the frequency. This can be represented, for the different regimes, by the fit

$\Delta v_{\mathrm{as}}=(1+\zeta) \Delta v_{\mathrm{obs}}$,

with

$$
\begin{aligned}
& \left.\zeta=\frac{0.57}{n_{\max }} \quad \text { (main-sequence regime: } n_{\max } \geq 15\right), \\
& \left.\zeta=0.038 \quad \text { (red giant regime: } n_{\max } \leq 15\right) .
\end{aligned}
$$

The consequence of these relations is examined in Sect. 4.2. As for the curvature, the justification of the two different regimes is based on the analysis of the offset $\varepsilon_{\text {obs }}$.

\subsection{Observed and asymptotic offsets}

The offsets $\varepsilon_{\mathrm{obs}}$ and $\varepsilon_{\mathrm{as}}$ are plotted in Figs. 7 and 8 . The fit of $\varepsilon_{\mathrm{obs}}$, initially given by Mosser et al. (2011) for red giants and updated by Corsaro et al. (2012), is prolonged to mainsequence stars with a nearly constant fit at $\varepsilon_{\mathrm{obs}, \mathrm{MS}} \simeq 1.4$. We based this fit on low-mass $G$ stars in order to avoid the more complex spectra of F stars that can be affected by the HD 49933 misidentification syndrome (Appourchaux et al. 2008; Benomar et al. 2009). The spread of $\varepsilon_{\mathrm{as}}$ is large since the propagation of the uncertainties from $\alpha_{\text {obs }}$ to $\varepsilon_{\text {as }}$ yields a large uncertainty: $\delta \varepsilon_{\mathrm{as}} \simeq 2 \delta \alpha_{\mathrm{obs}} / \alpha_{\mathrm{obs}} \simeq 0.4$.

We note in particular that the two different regimes seen for $\alpha_{\text {obs }}$ correspond to the different variations of $\varepsilon_{\text {obs }}$ with stellar evolution. The comparison between $\varepsilon_{\text {as }}$ and $\varepsilon_{\text {obs }}$ allows us to derive significant features. The regime where $\varepsilon_{\mathrm{obs}}$ does not change with $\Delta v_{\text {obs }}$ coincides with the regime where the curvature evolves with $n_{\max }^{-2}$. The correction provided by Eq. (13) then mainly corresponds to the constant term $2 a_{\mathrm{MS}}$. As a consequence, for lowmass main-sequence and subgiant stars the asymptotic value is very close to $1 / 4$ (Eq. (8), as found by Tassoul 1980). In the red-giant regime, the curvature varying as $n_{\max }^{-1}$ provides a variable correction, so that $\varepsilon_{\mathrm{as}}$ is close to $1 / 4$ also even if $\varepsilon_{\mathrm{obs}}$ varies with $\Delta v_{\text {obs }}$.

\subsection{Mass dependence}

For low-mass stars, the fact that we find a mean value $\left\langle\varepsilon_{\text {as }}\right\rangle$ of about $1 / 4$ suggests that the asymptotic expansion is valid for 

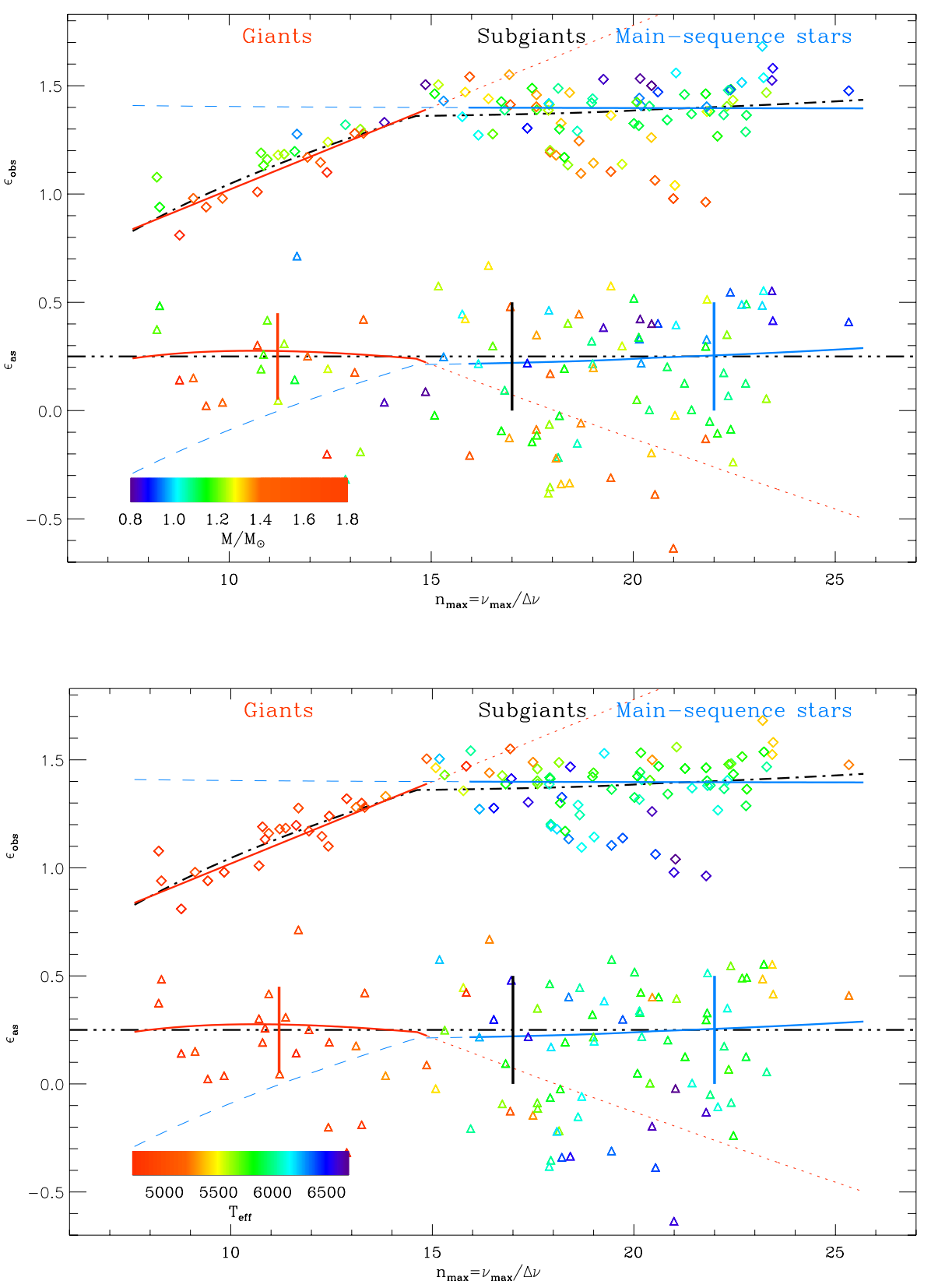

Fig. 7. Same as Fig. 4, for the observed (diamonds) and asymptotic (triangles) offsets. Both parameters are fitted, with dotted lines in the red giant regime and dashed lines in the main-sequence regime; thicker lines indicate the domain of validity of the fits. The triple-dotdashed line represents the Tassoul value $\varepsilon_{\text {as }}=$ $1 / 4$, and the dot-dashed line is the model of $\varepsilon_{\mathrm{obs}}$ (varying with $\log \Delta v_{\mathrm{obs}}$ in the red giant regime, and constant for less-evolved stars).
Fig. 8. Same as Fig. 7, with a color code depending on the effective temperature. describing solar-like oscillation spectra in a coherent way. This validity is also confirmed for red giants. For those stars, we may assume that $\varepsilon_{\mathrm{as}} \equiv 1 / 4$. Having the observed value $\varepsilon_{\mathrm{obs}}$ much larger than $\varepsilon_{\mathrm{as}}$ can be seen as an artefact of the curvature $\alpha_{\mathrm{obs}}$ introduced by the use of $\Delta v_{\mathrm{obs}}$ :

$\varepsilon_{\mathrm{obs}} \simeq \frac{1}{4}\left(1+n_{\max } \frac{\alpha_{\mathrm{obs}}}{2}\right)+n_{\mathrm{max}}^{2} \alpha_{\mathrm{obs}}$

However, we note in Fig. 7 a clear gradient of $\varepsilon_{\text {as }}$ with the stellar mass: high-mass stars have in general lower $\varepsilon_{\text {as }}$ than lowmass stars, similar to what is observed for $\varepsilon_{\mathrm{obs}}$. In massive stars, the curvature is more pronounced; $\varepsilon_{\mathrm{obs}}$ and $\varepsilon_{\mathrm{as}}$ are lower than in low-mass stars. As a consequence, the asymptotic value $\varepsilon_{\text {as }}$ cannot coincide with $1 / 4$, as is approximately the case for low-mass stars. We tried to reconcile this different behavior by taking into account a mass dependence in the fit of the curvature. In fact, fitting the higher curvature of high-mass stars would translate into a larger correction, in absolute value, from $\varepsilon_{\text {obs }}$ to $\varepsilon_{\text {as }}$, so that it cannot account for the difference.

We are therefore left with the conclusion that, contrary to low-mass stars, the asymptotic expansion is less satisfactory for describing the radial-mode oscillation spectra of high-mass stars. At this stage, we may imagine that in fact some features are superimposed on the asymptotic spectrum, such as signatures of glitches with longer period than the radial-order range where the radial modes are observed. Such long-period glitches can be due to the convective core in main-sequence stars with a mass larger than $1.2 M_{\odot}$; they are discussed in Sect. 4.1.

\section{Discussion}

We explore here some consequences of fitting the observed oscillation spectra with the exact asymptotic relation. First, we examine the possible limitations of this relation as defined by 
Tassoul (1980). Since the observed and asymptotic values of the large separation differ, directly extracting the stellar radius or mass from the measured value $\Delta v_{\mathrm{obs}}$ induces a non-negligible bias. Thus, we revisit the scaling relations which provide estimates of the stellar mass and radius. In a next step, we investigate the meaning of the term $\varepsilon_{\mathrm{obs}}$. We finally discuss the consequence of having $\varepsilon_{\text {as }}$ exactly equal to $1 / 4$. This assumption would make the asymptotic expansion useful for analyzing acoustic glitches. Demonstrating that radial modes are in all cases based on $\varepsilon_{\text {as }} \equiv 1 / 4$ will require some modeling, which is beyond the scope of this paper.

\subsection{Contribution of the glitches}

Solar and stellar oscillation spectra show that the asymptotic expansion is not enough for describing the low-degree oscillation pattern. Acoustic glitches yield significant modulation (e.g., Mazumdar et al. 2012, and references therein).

Defining the global curvature is not an easy task, since the radial oscillation is modulated by the glitches. With the asymptotic description of the signature of the glitch proposed by Provost et al. (1993), the asymptotic modulation adds a contribution $\delta v_{n, 0}$ to the eigenfrequency $v_{n, 0}$ defined by Eqs. (1) and (6), which can be written at first order:

$\frac{\delta v_{n, 0}}{\Delta v_{\mathrm{as}}}=\beta \sin 2 \pi \frac{n-n_{\mathrm{g}}}{N_{\mathrm{g}}}$,

where $\beta$ measures the amplitude of the glitch, $n_{\mathrm{g}}$ its phase, and $N_{\mathrm{g}}$ its period. This period varies as the ratio of the stellar acoustic radius divided by the acoustic radius at the discontinuity. Hence, deep glitches induce long-period modulation, whereas glitches in the upper stellar envelope have short periods. The phase $n_{\mathrm{g}}$ has no simple expression. The observed large separations vary approximately as

$$
\frac{\Delta v_{n, 0}}{\Delta v_{\text {obs }}} \simeq 1+\alpha_{\text {obs }}\left(n-n_{\max }\right)+\frac{2 \pi \beta}{N_{\mathrm{g}}} \cos 2 \pi \frac{n-n_{\mathrm{g}}}{N_{\mathrm{g}}},
$$

according to the derivation of Eq. (23). In the literature, we see typical values of $N_{\mathrm{g}}$ in the range 6-12, or even larger if the cause of the glitch is located in deep layers. The amplitude of the modulation represents a few percent of $\Delta v_{\text {obs }}$, so that the modulation term $2 \pi \beta / N_{\mathrm{g}}$ can greatly exceed the curvature $\alpha_{\mathrm{obs}}$. This explains the noisy aspect of Figs. 4 to 7 , which is due to larger spreads than the mean curvature. From $\varepsilon_{\text {as }}=1 / 4$, one should get $\varepsilon_{\text {obs,as }} \simeq 1.39$ for main-sequence stars. If the observed value $\varepsilon_{\mathrm{obs}}$ differs from the expected asymptotic observed $\varepsilon_{\mathrm{obs} \text {,as }}$, then one has to suppose that glitches explain the difference. The departure from $\varepsilon_{\mathrm{as}}=1 / 4$ of main-sequence stars with a larger mass than $1.2 M_{\odot}$ is certainly related to the influence of their convective core. If the period is long enough compared to the number of observable modes, it can translate into an apparent frequency offset, which is interpreted as an offset in $\varepsilon_{\text {obs }}$. Similarly, glitches due to the high contrast density between the core and the envelope in red giants, with a different phase according to the evolutionary status, might modify differently the curvature of their oscillation spectra. These hypotheses will be tested in a forthcoming work.

\subsection{Scaling relations revisited}

The importance of the measurements of $\Delta v_{\mathrm{obs}}$ and $v_{\max }$ is emphasized by their ability to provide relevant estimates of the stellar mass and radius:

$$
\begin{aligned}
& \frac{R_{\mathrm{obs}}}{R_{\odot}}=\left(\frac{v_{\max }}{v_{\max , \odot}}\right)\left(\frac{\Delta v_{\mathrm{obs}}}{\Delta v_{\odot}}\right)^{-2}\left(\frac{T_{\mathrm{eff}}}{T_{\odot}}\right)^{1 / 2} \\
& \frac{M_{\mathrm{obs}}}{M_{\odot}}=\left(\frac{v_{\max }}{v_{\max , \odot}}\right)^{3}\left(\frac{\Delta v_{\mathrm{obs}}}{\Delta v_{\odot}}\right)^{-4}\left(\frac{T_{\mathrm{eff}}}{T_{\odot}}\right)^{3 / 2}
\end{aligned}
$$

The solar values chosen as references are not fixed uniformly in the literature. Usually, internal calibration is ensured by the analysis of the solar low-degree oscillation spectrum with the same tool used for the asteroseismic spectra. Therefore, we used $\Delta v_{\odot}=135.5 \mu \mathrm{Hz}$ and $v_{\max , \odot}=3050 \mu \mathrm{Hz}$. One can find significantly different values, e.g., $\Delta v_{\odot}=134.9 \mu \mathrm{Hz}$ and $v_{\max , \odot}=$ $3120 \mu \mathrm{Hz}$ (Kallinger et al. 2010). We will see later that this diversity is not an issue if coherence and proper calibration are ensured.

These relations rely on the definition of the large separation, which scales as the square root of the mean stellar density (Eddington 1917), and $v_{\max }$, which scales as the cutoff acoustic frequency (Belkacem et al. 2011). From the observed value $\Delta v_{\mathrm{obs}}$, one gets biased estimates $R\left(\Delta v_{\mathrm{obs}}\right)$ and $M\left(\Delta v_{\mathrm{obs}}\right)$, since $\Delta v_{\mathrm{obs}}$ is underestimated when compared to $\Delta v_{\mathrm{as}}$. Taking into account the correction provided by Eq. (11), the corrected values are

$R_{\mathrm{as}} \simeq(1-2 \zeta) R_{\mathrm{obs}}$ and $M_{\mathrm{as}} \simeq(1-4 \zeta) M_{\mathrm{obs}}$,

with $\zeta$ defined by Eqs. (20) or (21), depending on the regime. The amplitude of the correction $\zeta$ can be as high as $3.8 \%$, but one must take into account the fact that scaling relations are calibrated on the Sun, so that one has to deduce the solar correction $\zeta_{\odot} \simeq 2.6 \%$. As a result, for subgiants and main-sequence stars, the systematic negative correction reaches about $5 \%$ for the seismic estimate of the mass and about $2.5 \%$ for the seismic estimate of the radius. The absolute corrections are maximum in the red giant regime. This justifies the correcting factors introduced for deriving masses and radii for CoRoT red giants (Eqs. (9) and (10) of Mosser et al. 2010), obtained by comparison with the modeling of red giants chosen as reference.

We checked the bias in an independent way by comparing the stellar masses obtained by modeling (sublist of 55 stars in Table 1) with the seismic masses. These seismic estimates appeared to be systematically $7.5 \%$ larger and had to be corrected. The comparison of the stellar radii yielded the same conclusion: the mean bias was of the order $2.5 \%$. Furthermore, we noted that the discrepancy increased significantly when the stellar radius increased, as expected from Eqs. (27), (20) and (21), since an increasing radius corresponds to a decreasing value of $n_{\max }$.

This implies that previous work based on the uncorrected scaling relations might be improved. This concerns ensemble asteroseismic results (e.g., Verner et al. 2011; Huber et al. 2011) or Galactic population analysis based on distance scaling (Miglio et al. 2009). This necessary systematic correction must be prepared by an accurate calibration of the scaling relations, taking into account the evolutionary status of the star (White et al. 2011; Miglio et al. 2012), cluster properties (Miglio et al. 2012), or independent interferometric measurements (Silva Aguirre et al. 2012).

At this stage, we suggest deriving the estimates of the stellar mass and radius from a new set of equations 
based on $\Delta v_{\mathrm{as}}$ or $\Delta v_{\mathrm{obs}}$ :

$\frac{R}{R_{\odot}}=\left(\frac{v_{\mathrm{max}}}{v_{\mathrm{ref}}}\right)\left(\frac{\Delta v}{\Delta v_{\mathrm{ref}}}\right)^{-2}\left(\frac{T_{\mathrm{eff}}}{T_{\odot}}\right)^{1 / 2}$

$\frac{M}{M_{\odot}}=\left(\frac{v_{\text {max }}}{v_{\text {ref }}}\right)^{3}\left(\frac{\Delta v}{\Delta v_{\text {ref }}}\right)^{-4}\left(\frac{T_{\mathrm{eff}}}{T_{\odot}}\right)^{3 / 2}$,

with new calibrated references $v_{\text {ref }}=3104 \mu \mathrm{Hz}$ and $\Delta v_{\text {ref }}=$ $138.8 \mu \mathrm{Hz}$ based on the comparison with the models of Table 1 . In case $\Delta v_{\text {obs }}$ is used, corrections provided by Eq. (27) must be applied; no correction is needed with the use of $\Delta v_{\text {as }}$. As expected from White et al. (2011), we noted that the quality of the estimates decreased for effective temperatures higher than $6500 \mathrm{~K}$ or lower than $5000 \mathrm{~K}$. We therefore limited the calibration to stars with a mass less than $1.3 M_{\odot}$. The accuracy of the fit for these stars is about $8 \%$ for the mass and $4 \%$ for the radius. Scaling relations are less accurate for $\mathrm{F}$ stars with higher mass and effective temperatures and for red giants. For those stars, the performance of the scaling relations is degraded by a factor 2 .

\subsection{Surface effect?}

\subsubsection{Interpretation of $\varepsilon_{\text {obs }}$}

We note that the scaling of $\varepsilon_{\text {obs }}$ for red giants as a function of $\log \Delta v$ implicitly or explicitly presented by many authors (Huber et al. 2010; Mosser et al. 2011; Kallinger et al. 2012; Corsaro et al. 2012) can in fact be explained by the simple consequence of the difference between $\Delta v_{\mathrm{obs}}$ and $\Delta v_{\mathrm{as}}$. Apart from the large spread, we derived that the mean value of $\varepsilon_{\text {as }}$, indicated by the dashed line in Fig. 7, is nearly a constant.

Following White et al. (2012), we examined how $\varepsilon_{\text {obs }}$ varies with effective temperature (Fig. 8). Unsurprizingly, we see the same trend for subgiants and main-sequence stars. Furthermore, there is a clear indication that a similar gradient is present in red giants. However, there is no similar trend for $\varepsilon_{\text {as }}$. This suggests that the physical reason for explaining the gradient of $\varepsilon_{\mathrm{obs}}$ with temperature is, in fact, firstly related to the stellar mass and not to the effective temperature.

The fact that $\varepsilon_{\mathrm{obs}}$ is very different from $\varepsilon_{\mathrm{as}}=1 / 4$ and varies with the large separation suggests that $\varepsilon_{\mathrm{obs}}$ cannot be seen solely as an offset relating the surface properties (e.g., White et al. 2012). In other words, $\varepsilon_{\text {obs }}$ should not be interpreted as a surface parameter, since its properties are closely related to the way the large separation is determined. Its value is also severely affected by the glitches. Any modulation showing a large period (in radial order) will translate into an additional offset that will be mixed with $\varepsilon_{\text {obs }}$.

In parallel, the mass dependence in $\varepsilon_{\text {as }}$ also indicates that it depends on more than surface properties. Examining the exact dependence of the offset $\varepsilon_{\text {as }}$ can be done by identifying it with the phase shift given by the eigenfrequency equation (Roxburgh \& Vorontsov 2000, 2001).

\subsubsection{Contribution of the upper atmosphere}

The uppermost part of the stellar atmosphere contributes to the slight modification of the oscillation spectrum, since the level of reflection of a pressure wave depends on its frequency (e.g., Mosser et al. 1994): the higher the frequency, the higher the level of reflection; the larger the resonant cavity, the smaller the apparent large separation. Hence, this effect gives rise to apparent variation of the observed large separations varying in the opposite direction compared to the effect demonstrated in this work. This effect was not considered in this work but must also be corrected for. It can be modeled when the contribution of photospheric layers is taken into account. For subgiants and main-sequence stars, its magnitude is of about $-0.04 \Delta v$ (Mathur et al. 2012), hence much smaller than the correction from $\Delta v_{\mathrm{obs}}$ to $\Delta v_{\mathrm{as}}$, which is of about $+1.14 \Delta v$ (Eq. (18)).

\subsection{A generic asymptotic relation}

We now make the assumption that $\varepsilon_{\text {as }}$ is strictly equal to $1 / 4$ for all solar-like oscillation patterns of low-mass stars and explore the consequences.

\subsubsection{Subgiants and main-sequence stars}

The different scalings between observed and asymptotic seismic parameters have indicated a dependence of the observed curvature close to $n_{\max }^{-2}$ for subgiants and main-sequence stars. As a result, it is possible to write the asymptotic relation for radial modes (Eq. (6)) as

$$
\begin{aligned}
\left.v_{n, 0}\right|_{\mathrm{MS}} & =\left(n+\varepsilon_{\mathrm{as}}+a_{\mathrm{MS}} \frac{n_{\mathrm{max}}}{n}\right) \Delta v_{\mathrm{as}} \\
& \simeq\left(n+\frac{1}{4}+0.57 \frac{n_{\max }}{n}\right) \Delta v_{\mathrm{as}} \\
& \simeq\left(n+\frac{1}{4}+\frac{12.8}{n}\left(\frac{M}{M_{\odot}} \frac{R_{\odot}}{R} \frac{T_{\odot}}{T_{\mathrm{eff}}}\right)^{1 / 2}\right) \Delta v_{\mathrm{as}} .
\end{aligned}
$$

This underlines the large similarity of stellar interiors. With such a development, the mean dimensionless value of the secondorder term is $a_{\mathrm{MS}}$, since the ratio $n_{\max } / n$ is close to 1 and does not vary with $n_{\max }$. However, compared to the dominant term in $n$, its relative value decreases when $n_{\max }$ increases. In absolute value, the second-order term scales as $\Delta v_{\text {as }} v_{\max } / v$, so that the dimensionless term $d_{1}$ of Eq. (1) is proportional to $v_{\max } / \Delta v_{\mathrm{as}}$, with a combined contribution of the stellar mean density $\left(\Delta v_{\text {as }}\right.$ term) and acoustic cutoff frequency ( $v_{\max }$, hence $\nu_{\mathrm{c}}$ contribution, Belkacem et al. 2011).

\subsubsection{Red giants}

For red giants, the asymptotic relation reduces to

$$
\begin{aligned}
\left.v_{n, 0}\right|_{\mathrm{RG}} & =\left(n+\varepsilon_{\mathrm{as}}+a_{\mathrm{RG}}^{\prime} \frac{n_{\mathrm{max}}^{2}}{n}\right) \Delta v_{\mathrm{as}} \\
& \simeq\left(n+\frac{1}{4}+0.037 \frac{n_{\mathrm{max}}^{2}}{n}\right) \Delta v_{\mathrm{as}} \\
& \simeq\left(n+\frac{1}{4}+\frac{18.3}{n}\left(\frac{M}{M_{\odot}} \frac{R_{\odot}}{R} \frac{T_{\odot}}{T_{\mathrm{eff}}}\right)\right) \Delta v_{\mathrm{as}},
\end{aligned}
$$

with $a_{\mathrm{RG}}^{\prime}=a_{\mathrm{RG}} /\left(1+a_{\mathrm{RG}}\right)$. The relationship given by Eq. (17) ensures that the relative importance of the second-order term saturates when $n_{\max }$ decreases. Its relative influence compared to the radial order is $a_{\mathrm{RG}}^{\prime}$, of about $4 \%$. Contrary to less-evolved stars, the second-order term for red giants is in fact proportional to $v_{\max }^{2} / v$ and is predominantly governed by the surface gravity. 


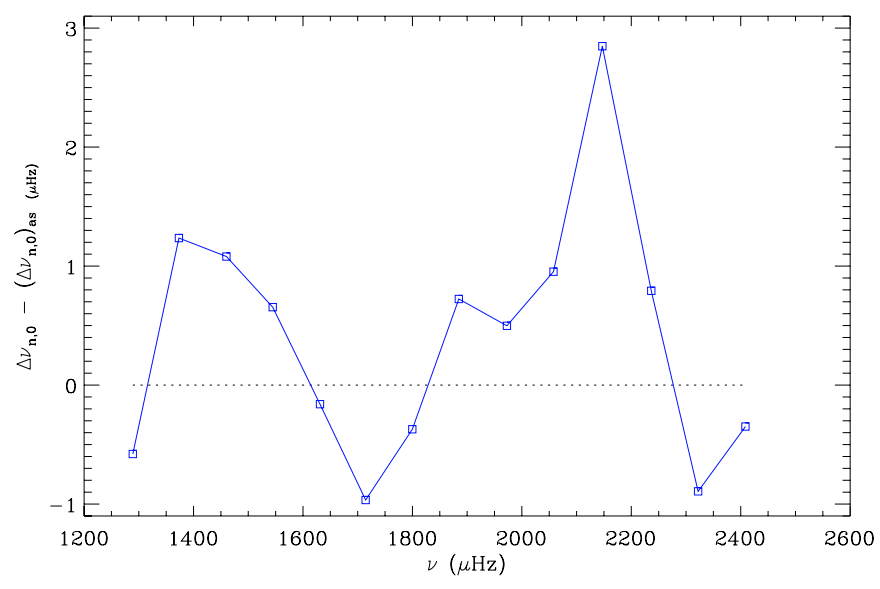

Fig. 9. Variation of the large separation difference $\Delta v_{n+1,0}-\left(\Delta v_{n, 0}\right)_{\text {as }}$ as a function of $v_{n, 0}$ for the star HD 49933.

\subsubsection{Measuring the glitches}

These discrepancies to the generic relations express, in fact, the variety of stars. In each regime, Eqs. (30) and (33) provide a reference for the oscillation pattern, and Eq. (23) indicates the small departure due to the specific interior properties of a star. Asteroseismic inversion could not operate if all oscillation spectra were degenerate and exactly similar to the mean asymptotic spectrum depicted by Eqs. (30) and (33). Conversely, if one assumes that the asymptotic relation provides a reliable reference case for a given stellar model represented by its large separation, then comparing an observed spectrum to the mean spectrum expected at $\Delta v$ may provide a way to determine the glitches. In other words, glitches may correspond to observed modulation after subtraction of the mean curvature. We show this in an example (Fig. 9), where we subtracted the mean asymptotic slope derived from Eq. (18) from the local large separations $v_{n+1,0}-v_{n, 0}$.

\section{Conclusion}

We have addressed some consequences of the expected difference between the observed and asymptotic values of the large separation. We derived the curvature of the radial-mode oscillation pattern in a large set of solar-like oscillation spectra from the variation in frequency of the spacings between consecutive radial orders. We then proposed a simple model to represent the observed spectra with the exact asymptotic form. Despite the spread of the data, the ability of the Tassoul asymptotic expansion to account for the solar-like oscillation spectra is confirmed, since we have demonstrated coherence between the observed and asymptotic parameters. Two regimes have been identified: one corresponds to the subgiants and main-sequence stars, the other to red giants. These regimes explain the variation of the observed offset $\varepsilon_{\text {obs }}$ with the large separation.

Curvature and second-order effect: we have verified that the curvature observed in the échelle diagram of solar-like oscillation spectra corresponds to the second-order term of the Tassoul equation. We have shown that the curvature scales approximately as $\left(\Delta v / v_{\max }\right)^{2}$ for subgiants and main-sequence stars. Its behavior changes in the red giant regime, where it varies approximately as $\left(\Delta v / v_{\max }\right)$ for red giants.
Large separation and scaling relations: as the ratio $\Delta v_{\mathrm{as}} / \Delta v_{\mathrm{obs}}$ changes along the stellar evolution (from about $2 \%$ for a lowmass dwarf to $4 \%$ for a giant), scaling relations must be corrected to avoid a systematic overestimate of the seismic proxies. Corrections are proposed for the stellar radius and mass, which avoid a bias of $2.5 \%$ for $R$ and $5 \%$ for $M$. The corrected and calibrated scaling relations then provide an estimate of $R$ and $M$ with 1- $\sigma$ uncertainties of, respectively, 4 and $8 \%$ for low-mass stars.

Offsets: the observed values $\varepsilon_{\text {obs }}$ are affected by the definition and the measurement of the large separation. Their spread is amplified by all glitches affecting solar-like oscillation spectra. We made clear that the variation of $\varepsilon_{\text {obs }}$ with stellar evolution is mainly an artefact due to the use of $\Delta v_{\text {obs }}$ instead of $\Delta v_{\mathrm{as}}$ in the asymptotic relation. This work shows that the asymptotic value $\varepsilon_{\text {as }}$ is a small constant, which does vary much throughout stellar evolution for low-mass stars and red giants. It is very close to the value $1 / 4$ derived from the asymptotic expansion for low-mass stars.

Generic asymptotic relation: according to this, we have established a generic form of the asymptotic relation of radial modes in low-mass stars. The mean oscillation pattern based on this relation can serve as a reference for rapidly analyzing a large amount of data for identifying unambiguously an oscillation pattern and for determining acoustic glitches. Departure from the generic asymptotic relation is expected for main-sequence stars with a convective core and for red giants, according to the asymptotic expansion of structure discontinuities.

Last but not least, this work implies that all the quantitative conclusions of previous analyses that have created confusion between $\Delta v_{\text {as }}$ and $\Delta v_{\text {obs }}$ have to be reconsidered. The observed values derived from the oscillation spectra have to be translated into asymptotic values before any physical analysis. To avoid confusion, it is also necessary to specify which value of the large separation is considered. A coherent notation for the observed value of the large separation at $v_{\max }$ should be $\Delta v_{\max }$.

\section{References}

Appourchaux, T., Michel, E., Auvergne, M., et al. 2008, A\&A, 488, 705 Appourchaux, T., Chaplin, W. J., García, R. A., et al. 2012, A\&A, 543, A54 Audard, N., \& Provost, J. 1994, A\&A, 282, 73

Ballot, J., Gizon, L., Samadi, R., et al. 2011, A\&A, 530, A97

Barban, C., Deheuvels, S., Baudin, F., et al. 2009, A\&A, 506, 51

Baudin, F., Barban, C., Goupil, M. J., et al. 2012, A\&A, 538, A73

Bazot, M., Vauclair, S., Bouchy, F., \& Santos, N. C. 2005, A\&A, 440, 615

Bazot, M., Ireland, M. J., Huber, D., et al. 2011, A\&A, 526, L4

Bazot, M., Campante, T. L., Chaplin, W. J., et al. 2012, A\&A, 544, A106

Beck, P. G., Bedding, T. R., Mosser, B., et al. 2011, Science, 332, 205

Beck, P. G., Montalban, J., Kallinger, T., et al. 2012, Nature, 481, 55

Bedding, T. R., Butler, R. P., Kjeldsen, H., et al. 2001, ApJ, 549, L105

Bedding, T. R., Kjeldsen, H., Butler, R. P., et al. 2004, ApJ, 614, 380

Bedding, T. R., Huber, D., Stello, D., et al. 2010a, ApJ, 713, L176

Bedding, T. R., Kjeldsen, H., Campante, T. L., et al. 2010b, ApJ, 713, 935

Belkacem, K., Goupil, M. J., Dupret, M. A., et al. 2011, A\&A, 530, A142

Benomar, O., Baudin, F., Campante, T. L., et al. 2009, A\&A, 507, L13

Bouchy, F., Bazot, M., Santos, N. C., Vauclair, S., \& Sosnowska, D. 2005, A\&A, 440,609

Bruntt, H., Basu, S., Smalley, B., et al. 2012, MNRAS, 423, 122

Campante, T. L., Handberg, R., Mathur, S., et al. 2011, A\&A, 534, A6

Carrier, F., De Ridder, J., Baudin, F., et al. 2010, A\&A, 509, A73

Catala, C., Forveille, T., \& Lai, O. 2006, AJ, 132, 2318

Christensen-Dalsgaard, J., \& Frandsen, S. 1983, Sol. Phys., 82, 469

Christensen-Dalsgaard, J., \& Perez Hernandez, F. 1992, MNRAS, 257, 62

Corsaro, E., Stello, D., Huber, D., et al. 2012, ApJ, 757, 190

Creevey, O. L., Doğan, G., Frasca, A., et al. 2012, A\&A, 537, A111 
De Ridder, J., Barban, C., Baudin, F., et al. 2009, Nature, 459, 398 Deheuvels, S., \& Michel, E. 2011, A\&A, 535, A91

Deheuvels, S., Bruntt, H., Michel, E., et al. 2010, A\&A, 515, A87

Deheuvels, S., García, R. A., Chaplin, W. J., et al. 2012, ApJ, 756, 19

di Mauro, M. P., Cardini, D., Catanzaro, G., et al. 2011, MNRAS, 415, 3783

Eddington, A. S. 1917, The Observatory, 40, 290

Gaulme, P., Deheuvels, S., Weiss, W. W., et al. 2010, A\&A, 524, A47

Grec, G., Fossat, E., \& Pomerantz, M. A. 1983, Sol. Phys., 82, 55

Hekker, S., Kallinger, T., Baudin, F., et al. 2009, A\&A, 506, 465

Hekker, S., Elsworth, Y., De Ridder, J., et al. 2011, A\&A, 525, A131

Hekker, S., Elsworth, Y., Mosser, B., et al. 2012, A\&A, 544, A90

Howell, S. B., Rowe, J. F., Bryson, S. T., et al. 2012, ApJ, 746, 123

Huber, D., Bedding, T. R., Stello, D., et al. 2010, ApJ, 723, 1607

Huber, D., Bedding, T. R., Stello, D., et al. 2011, ApJ, 743, 143

Huber, D., Ireland, M. J., Bedding, T. R., et al. 2012, ApJ, 760, 32

Jiang, C., Jiang, B. W., Christensen-Dalsgaard, J., et al. 2011, ApJ, 742, 120

Kallinger, T., Mosser, B., Hekker, S., et al. 2010, A\&A, 522, A1

Kallinger, T., Hekker, S., Mosser, B., et al. 2012, A\&A, 541, A51

Kjeldsen, H., Bedding, T. R., Butler, R. P., et al. 2005, ApJ, 635, 1281

Mathur, S., Handberg, R., Campante, T. L., et al. 2011, ApJ, 733, 95

Mathur, S., Metcalfe, T. S., Woitaszek, M., et al. 2012, ApJ, 749, 152

Mathur, S., Bruntt, H., Catala, C., et al. 2013, A\&A, 549, A12

Mazumdar, A., Michel, E., Antia, H. M., \& Deheuvels, S. 2012, A\&A, 540, A31

Metcalfe, T. S., Chaplin, W. J., Appourchaux, T., et al. 2012, ApJ, 748, L10

Michel, E., Baglin, A., Auvergne, M., et al. 2008, Science, 322, 558

Miglio, A., Montalbán, J., Baudin, F., et al. 2009, A\&A, 503, L21

Miglio, A., Montalbán, J., Carrier, F., et al. 2010, A\&A, 520, L6
Miglio, A., Brogaard, K., Stello, D., et al. 2012, MNRAS, 419, 2077 Mosser, B., \& Appourchaux, T. 2009, A\&A, 508, 877

Mosser, B., Gautier, D., Schmider, F. X., \& Delache, P. 1991, A\&A, 251, 356

Mosser, B., Gudkova, T., \& Guillot, T. 1994, A\&A, 291, 1019

Mosser, B., Bouchy, F., Martić, M., et al. 2008a, A\&A, 478, 197

Mosser, B., Deheuvels, S., Michel, E., et al. 2008b, A\&A, 488, 635

Mosser, B., Michel, E., Appourchaux, T., et al. 2009, A\&A, 506, 33

Mosser, B., Belkacem, K., Goupil, M., et al. 2010, A\&A, 517, A22

Mosser, B., Belkacem, K., Goupil, M., et al. 2011, A\&A, 525, L9

Mosser, B., Elsworth, Y., Hekker, S., et al. 2012a, A\&A, 537, A30

Mosser, B., Goupil, M. J., Belkacem, K., et al. 2012b, A\&A, 548, A10

Mosser, B., Goupil, M. J., Belkacem, K., et al. 2012c, A\&A, 540, A143

Provost, J., Mosser, B., \& Berthomieu, G. 1993, A\&A, 274, 595

Reese, D. R., Marques, J. P., Goupil, M. J., Thompson, M. J., \& Deheuvels, S. 2012, A\&A, 539, A63

Roxburgh, I. W., \& Vorontsov, S. V. 2000, MNRAS, 317, 141

Roxburgh, I. W., \& Vorontsov, S. V. 2001, MNRAS, 322, 85

Scherrer, P. H., Wilcox, J. M., Christensen-Dalsgaard, J., \& Gough, D. O. 1983, Sol. Phys., 82, 75

Silva Aguirre, V., Casagrande, L., Basu, S., et al. 2012, ApJ, 757, 99

Stello, D., Basu, S., Bruntt, H., et al. 2010, ApJ, 713, L182

Stello, D., Huber, D., Kallinger, T., et al. 2011, ApJ, 737, L10

Tassoul, M. 1980, ApJS, 43, 469

Thévenin, F., Provost, J., Morel, P., et al. 2002, A\&A, 392, L9

Verner, G. A., Elsworth, Y., Chaplin, W. J., et al. 2011, MNRAS, 415, 3539

White, T. R., Bedding, T. R., Stello, D., et al. 2011, ApJ, 743, 161

White, T. R., Bedding, T. R., Gruberbauer, M., et al. 2012, ApJ, 751, L36 
Table 1. Stellar parameters.

\begin{tabular}{|c|c|c|c|c|c|c|c|c|c|c|c|c|}
\hline $\operatorname{Star}^{a}$ & $n_{\max }$ & $\begin{array}{l}\Delta v_{\mathrm{obs}} \\
(\mu \mathrm{Hz})\end{array}$ & $\begin{array}{l}\Delta v_{\mathrm{as}}^{b} \\
(\mu \mathrm{Hz})\end{array}$ & $\begin{array}{c}v_{\max } \\
(\mu \mathrm{Hz})\end{array}$ & $\varepsilon_{\mathrm{obs}}$ & $\begin{array}{r}\alpha_{\text {obs }} \\
\times 10^{3} \\
\end{array}$ & $\begin{array}{r}T_{\text {eff }^{c}} \\
(\mathrm{~K})\end{array}$ & $\begin{array}{c}M_{\text {mod }}{ }^{d} \\
\left(M_{\odot}\right)\end{array}$ & $\begin{array}{c}M^{e} \\
\left(M_{\odot}\right)\end{array}$ & $\begin{array}{c}R_{\bmod }{ }^{d} \\
\left(R_{\odot}\right)\end{array}$ & $\begin{array}{c}R^{e} \\
\left(R_{\odot}\right)\end{array}$ & Ref. $^{f}$ \\
\hline HD 181907 & 8.2 & 3.41 & 3.55 & 28.0 & 1.08 & 10.2 & 4790 & 1.20 & 1.29 & 12.20 & 12.6 & 2010Car, 2010Mig \\
\hline KIC 4044238 & 8.3 & 4.07 & 4.23 & 33.7 & 0.94 & 6.5 & 4800 & & 1.11 & & 10.6 & 2012Mos \\
\hline HD 50890 & 8.8 & 1.71 & 1.78 & 15.0 & 0.81 & 8.6 & 4670 & 4.20 & 3.03 & 29.90 & 26.4 & 2012Bau \\
\hline KIC 5000307 & 9.1 & 4.74 & 4.93 & 43.2 & 0.98 & 9.9 & 4992 & & 1.35 & & 10.2 & 2012Mos \\
\hline KIC 9332840 & 9.4 & 4.39 & 4.57 & 41.4 & 0.94 & 10.3 & 4847 & & 1.55 & & 11.3 & 2012Mos \\
\hline KIC 4770846 & 9.8 & 5.48 & 5.70 & 53.9 & 0.98 & 9.7 & 4801 & & 1.39 & & 9.37 & $2012 \mathrm{Mo} 2$ \\
\hline KIC 2013502 & 10.7 & 5.72 & 5.95 & 61.2 & 1.01 & 6.1 & 4835 & & 1.73 & & 9.80 & 2012Mos \\
\hline KIC 10866415 & 10.8 & 8.75 & 9.10 & 94.4 & 1.19 & 8.5 & 4812 & & 1.15 & & 6.44 & $2012 \mathrm{Mo} 2$ \\
\hline KIC 11550492 & 10.9 & 8.70 & 9.05 & 94.4 & 1.13 & 7.3 & 4723 & & 1.14 & & 6.46 & 2012Mo2 \\
\hline KIC 9574650 & 10.9 & 9.64 & 10.03 & 105 & 1.16 & 6.1 & 5015 & & 1.16 & & 6.06 & 2012Mo2 \\
\hline KIC 3744043 & 11.2 & 9.90 & 10.30 & 111 & 1.18 & 9.0 & 4994 & & 1.21 & & 6.03 & 2012Mos \\
\hline KIC 9267654 & 11.4 & 10.4 & 10.8 & 117 & 1.18 & 6.7 & 4965 & & 1.19 & & 5.83 & $2012 \mathrm{Mo} 2$ \\
\hline KIC 6144777 & 11.6 & 11.0 & 11.5 & 128 & 1.20 & 7.8 & 4657 & & 1.09 & & 5.42 & $2012 \mathrm{Mo} 2$ \\
\hline KIC 5858947 & 11.7 & 14.5 & 15.1 & 169 & 1.28 & 4.0 & 4977 & & 0.92 & & 4.27 & $2012 \mathrm{Mo} 2$ \\
\hline KIC 6928997 & 11.9 & 10.1 & 10.5 & 120 & 1.17 & 6.4 & 4800 & & 1.35 & & 6.20 & 2011Bec, 2012Mos \\
\hline KIC 11618103 & 12.3 & 9.38 & 9.76 & 115 & 1.15 & 10.0 & 4870 & 1.45 & 1.60 & 6.60 & 6.87 & 2011Jia \\
\hline KIC 8378462 & 12.4 & 7.27 & 7.56 & 90.3 & 1.10 & 8.5 & 4962 & & 2.21 & & 9.06 & 2012Mos \\
\hline KIC 12008916 & 12.4 & 12.9 & 13.4 & 159 & 1.24 & 6.7 & 4830 & 1.26 & 1.21 & 5.18 & 5.07 & 2012Bec \\
\hline KIC 9882316 & 13.1 & 13.7 & 14.2 & 179 & 1.28 & 6.4 & 5228 & & 1.49 & & 5.22 & 2012Mos \\
\hline KIC 5356201 & 13.2 & 15.8 & 16.5 & 209 & 1.30 & 8.6 & 4840 & 1.23 & 1.19 & 4.47 & 4.38 & 2012Bec \\
\hline KIC 8366239 & 13.3 & 13.7 & 14.2 & 182 & 1.28 & 4.8 & 4980 & 1.49 & 1.46 & 5.30 & 5.18 & 2012Bec \\
\hline KIC 7341231 & 13.8 & 28.8 & 30.0 & 399 & 1.33 & 6.7 & 5300 & 0.83 & 0.85 & 2.62 & 2.63 & 2012Deh \\
\hline KIC 11717120 & 14.9 & 37.3 & 38.8 & 555 & 1.50 & 6.4 & 5150 & & 0.78 & & 2.15 & 2012App, 2012Bru \\
\hline KIC 9574283 & 15.1 & 29.7 & 30.9 & 448 & 1.46 & 6.5 & 5440 & & 1.11 & & 2.82 & 2012App \\
\hline KIC 8026226 & 15.2 & 34.3 & 35.6 & 520 & 1.50 & 4.0 & 6230 & & 1.21 & & 2.64 & 2012App, 2012Bru \\
\hline KIC 5607242 & 15.3 & 39.8 & 41.4 & 610 & 1.43 & 5.0 & 5680 & & 0.93 & & 2.18 & 2012App \\
\hline KIC 8702606 & 15.8 & 39.7 & 41.2 & 626 & 1.36 & 3.6 & 5540 & & 0.99 & & 2.23 & 2012App, 2012Bru \\
\hline KIC 4351319 & 15.8 & 24.4 & 25.4 & 387 & 1.47 & 4.1 & 4700 & 1.30 & 1.27 & 3.40 & 3.36 & 2011diM \\
\hline KIC 11771760 & 16.0 & 31.7 & 32.9 & 505 & 1.54 & 6.9 & 6030 & & 1.46 & & 2.96 & 2012App \\
\hline KIC 7976303 & 16.3 & 51.1 & 53.0 & 833 & 1.27 & 4.0 & 6260 & 1.17 & 1.02 & 2.03 & 1.91 & 2012App, 2012Mat \\
\hline HD 182736 & 16.4 & 34.6 & 35.9 & 568 & 1.44 & 2.8 & 5261 & 1.30 & 1.19 & 2.70 & 2.61 & $2012 \mathrm{Hub}$ \\
\hline KIC 10909629 & 16.5 & 49.2 & 51.0 & 813 & 1.28 & 3.5 & 6490 & & 1.17 & & 2.05 & 2012App \\
\hline KIC 11414712 & 16.7 & 43.6 & 45.2 & 730 & 1.43 & 5.4 & 5635 & & 1.11 & & 2.19 & 2012App, 2012Bru \\
\hline KIC 5955122 & 16.8 & 49.1 & 50.9 & 826 & 1.39 & 4.6 & 5837 & & 1.06 & & 1.99 & 2012App, 2012Bru \\
\hline KIC 7799349 & 16.9 & 33.1 & 34.2 & 560 & 1.55 & 5.9 & 5115 & & 1.31 & & 2.78 & 2012App, 2012Bru \\
\hline Procyon & 17.0 & 54.1 & 56.0 & 918 & 1.41 & 3.2 & 6550 & 1.46 & 1.17 & 2.04 & 1.93 & 2010Bed, 2008Mos \\
\hline KIC 1435467 & 17.4 & 79.6 & 82.4 & 1384 & 1.30 & 3.6 & 6570 & & 0.86 & & 1.35 & 2012App, 2012Bru \\
\hline KIC 12508433 & 17.5 & 44.8 & 46.3 & 784 & 1.49 & 5.4 & 5280 & & 1.13 & & 2.16 & 2012App \\
\hline KIC 11193681 & 17.6 & 42.7 & 44.2 & 752 & 1.40 & 4.8 & 5690 & & 1.35 & & 2.37 & 2012App \\
\hline KIC 11395018 & 17.6 & 47.4 & 49.0 & 834 & 1.46 & 3.5 & 5650 & 1.35 & 1.20 & 2.21 & 2.13 & 2011Mat, 2012Cre \\
\hline KIC 11026764 & 17.6 & 50.3 & 52.0 & 885 & 1.39 & 4.9 & 5682 & & 1.14 & & 2.01 & 2011Cam, 2012App, 2012Bru \\
\hline HD 49385 & 17.9 & 55.5 & 57.4 & 994 & 1.39 & 5.6 & 6095 & 1.25 & 1.21 & 1.94 & 1.92 & 2010Deh, 2011Deh \\
\hline KIC 10920273 & 17.9 & 57.2 & 59.2 & 1026 & 1.41 & 4.6 & 5880 & 1.23 & 1.12 & 1.88 & 1.83 & 2011Cam, 2012Cre \\
\hline KIC 10018963 & 17.9 & 55.1 & 56.9 & 988 & 1.20 & 4.9 & 6020 & 1.17 & 1.21 & 1.91 & 1.93 & 2012App, 2012Bru \\
\hline KIC 3632418 & 17.9 & 60.4 & 62.4 & 1084 & 1.19 & 3.2 & 6190 & 1.40 & 1.15 & 1.91 & 1.78 & 2012App, 2012Sil, 2012Bru \\
\hline KIC 10162436 & 18.1 & 55.5 & 57.3 & 1004 & 1.18 & 4.3 & 6200 & 1.36 & 1.28 & 2.01 & 1.96 & 2012App, 2012Sil, 2012Bru \\
\hline KIC 11713510 & 18.1 & 68.9 & 71.2 & 1249 & 1.42 & 2.9 & 5930 & 1.00 & 0.97 & 1.57 & 1.54 & 2012Mat \\
\hline KIC 8524425 & 18.1 & 59.4 & 61.4 & 1078 & 1.49 & 5.2 & 5634 & & 1.05 & & 1.75 & 2012App, 2012Bru \\
\hline KIC 7747078 & 18.2 & 53.8 & 55.5 & 977 & 1.30 & 4.0 & 5840 & 1.13 & 1.23 & 1.95 & 1.97 & 2012App, 2012Sil, 2012Bru \\
\hline KIC 7103006 & 18.2 & 58.9 & 60.8 & 1072 & 1.33 & 5.1 & 6394 & & 1.30 & & 1.89 & 2012App, 2012Bru \\
\hline HD 169392 & 18.3 & 56.3 & 58.1 & 1030 & 1.17 & 2.9 & 5850 & 1.15 & 1.20 & 1.88 & 1.90 & $2012 \mathrm{Ma} 2$ \\
\hline
\end{tabular}

Notes. ${ }^{(a)}$ Stars are sorted by increasing $n_{\max }$ values. ${ }^{(b)} \Delta v_{\text {as }}$ is derived from this work. ${ }^{(c)} T_{\text {eff }}$ provided by Bruntt et al. (2012), when available. ${ }^{(d)} M_{\text {mod }}$ and $R_{\text {mod }}$ were obtained from modeling or from interferometry, not from seismic scaling relations. ${ }^{(e)} M$ and $R$ are derived from the scaling relations Eqs. (28) and (29) with the new calibrations defined by this work.

References. 2002The = Thévenin et al. (2002); 2004Bed = Bedding et al. (2004); 2005Baz = Bazot et al. (2005); 2005Bou = Bouchy et al. (2005); $2005 \mathrm{Kje}=$ Kjeldsen et al. (2005); 2006Cat = Catala et al. (2006); 2008Mos = Mosser et al. (2008a); 2009Bar = Barban et al. $(2009) ; 2009 \mathrm{Ben}=$ Benomar et al. (2009); 2010Bed = Bedding et al. (2010b); 2010Car = Carrier et al. (2010); 2010Deh = Deheuvels et al. (2010); 2010Gau = Gaulme et al. (2010); 2010Mig = Miglio et al. (2010); 2011Bal = Ballot et al. (2011); 2011Baz = Bazot et al. (2011); 2011Bec = Beck et al. (2011); 2011Cam = Campante et al. (2011); 2011Deh = Deheuvels \& Michel (2011); 2011diM = di Mauro et al. (2011); 2011Jia = Jiang et al. (2011); 2011Mat = Mathur et al. (2011); 2012App = Appourchaux et al. (2012); 2012Bau = Baudin et al. (2012); 2012Baz = Bazot et al. (2012); 2012Bec = Beck et al. (2012); 2012Bru = Bruntt et al. (2012); 2012Cre = Creevey et al. (2012); 2012Deh = Deheuvels et al. (2012); 2012Giz = Gizon et al. (2012); 2012How = Howell et al. (2012); 2012Hub = Huber et al. (2012); 2012Mat = Mathur et al. (2012); 2012Ma2 = Mathur et al. (2013); 2012Met = Metcalfe et al. (2012); 2012Mos = Mosser et al. (2012c); 2012Mo2 = Mosser et al. (2012b); 2012Oze = Ozel et al. (2012); 2012Ree = Reese et al. (2012); 2012Sil = Silva Aguirre et al. (2012). 
Table 1. continued.

\begin{tabular}{|c|c|c|c|c|c|c|c|c|c|c|c|c|}
\hline $\operatorname{Star}^{a}$ & $n_{\max }$ & $\begin{array}{l}\Delta v_{\text {obs }} \\
(\mu \mathrm{Hz})\end{array}$ & $\begin{array}{l}\Delta v^{a b}{ }^{b} \\
(\mu \mathrm{Hz})\end{array}$ & $\begin{array}{c}v_{\max } \\
(\mu \mathrm{Hz})\end{array}$ & $\varepsilon_{\mathrm{obs}}$ & $\begin{array}{r}\alpha_{\text {obs }} \\
\times 10^{3}\end{array}$ & $\begin{array}{c}T_{\text {eff }^{c}} \\
(\mathrm{~K})\end{array}$ & $\begin{array}{c}M_{\text {mod }}{ }^{d} \\
\left(M_{\odot}\right)\end{array}$ & $\begin{array}{c}M^{e} \\
\left(M_{\odot}\right)\end{array}$ & $\begin{array}{c}R_{\text {mod }}{ }^{d} \\
\left(R_{\odot}\right)\end{array}$ & $\begin{array}{c}R^{e} \\
\left(R_{\odot}\right)\end{array}$ & Ref. $^{f}$ \\
\hline KIC 9812850 & 18.4 & 64.5 & 66.6 & 1186 & 1.13 & 2.1 & 6325 & & 1.20 & & 1.73 & 2012App, 2012Bru \\
\hline KIC 12317678 & 18.4 & 63.1 & 65.1 & 1162 & 1.47 & 5.4 & 6540 & & 1.30 & & 1.80 & 2012App \\
\hline KIC 8694723 & 18.6 & 74.3 & 76.7 & 1384 & 1.29 & 4.2 & 6120 & & 1.03 & & 1.50 & 2012App, 2012Bru \\
\hline KIC 8228742 & 18.7 & 61.8 & 63.8 & 1153 & 1.25 & 2.3 & 6042 & 1.31 & 1.22 & 1.84 & 1.79 & 2012Mat, 2012Sil, 2012Bru \\
\hline KIC 10273246 & 18.7 & 48.8 & 50.4 & 913 & 1.10 & 3.3 & 6150 & 1.37 & 1.60 & 2.19 & 2.30 & 2011Cam, 2012Cre \\
\hline KIC 6933899 & 19.0 & 71.8 & 74.1 & 1362 & 1.42 & 3.0 & 5860 & 1.10 & 1.06 & 1.58 & 1.55 & 2012Mat, 2012Bru \\
\hline KIC 11244118 & 19.0 & 71.2 & 73.4 & 1352 & 1.44 & 3.4 & 5745 & 1.01 & 1.04 & 1.55 & 1.55 & 2012Mat, 2012Bru \\
\hline HD 179070 & 19.0 & 60.6 & 62.6 & 1153 & 1.14 & 2.6 & 6131 & 1.34 & 1.35 & 1.86 & 1.88 & 2012How \\
\hline KIC 9410862 & 19.3 & 105.6 & 108.9 & 2034 & 1.53 & 3.1 & 6180 & & 0.82 & & 1.10 & 2012App \\
\hline KIC 10355856 & 19.4 & 67.0 & 69.1 & 1303 & 1.10 & 3.8 & 6350 & & 1.38 & & 1.77 & 2012App, 2012Bru \\
\hline KIC 12258514 & 19.4 & 74.5 & 76.8 & 1449 & 1.36 & 2.0 & 5930 & 1.22 & 1.12 & 1.59 & 1.54 & 2012Mat, 2012Sil, 2012Bru \\
\hline KIC 7206837 & 19.7 & 78.9 & 81.3 & 1556 & 1.14 & 2.1 & 6384 & & 1.24 & & 1.53 & 2012App, 2012Bru \\
\hline KIC 10516096 & 20.0 & 84.4 & 86.9 & 1689 & 1.33 & 2.0 & 5940 & 1.12 & 1.09 & 1.42 & 1.40 & 2012Mat, 2012Bru \\
\hline KIC 7680114 & 20.1 & 84.9 & 87.4 & 1705 & 1.42 & 3.4 & 5855 & 1.19 & 1.07 & 1.45 & 1.39 & 2012Mat, 2012Bru \\
\hline KIC 8760414 & 20.2 & 116.4 & 119.9 & 2349 & 1.53 & 2.7 & 5787 & 0.81 & 0.78 & 1.02 & 1.01 & 2012Mat, 2012App, 2012Bru \\
\hline KIC 3656476 & 20.4 & 93.3 & 96.1 & 1904 & 1.41 & 3.4 & 5710 & 1.09 & 0.98 & 1.32 & 1.27 & 2012Mat, 2012Bru \\
\hline KIC 11081729 & 20.4 & 89.0 & 91.6 & 1820 & 1.26 & 3.5 & 6630 & & 1.30 & & 1.44 & 2012App, 2012Bru \\
\hline HD 46375 & 20.5 & 154.0 & 158.5 & 3150 & 1.50 & 2.6 & 5300 & & 0.54 & & 0.74 & 2010Gau \\
\hline KIC 9139163 & 20.5 & 80.3 & 82.6 & 1649 & 1.06 & 3.5 & 6400 & 1.40 & 1.38 & 1.57 & 1.57 & 2012App, 2012Sil, 2012Bru \\
\hline KIC 9098294 & 20.6 & 108.7 & 111.9 & 2241 & 1.47 & 2.5 & 5840 & & 0.90 & & 1.11 & 2012App, 2012Bru \\
\hline KIC 4914923 & 20.8 & 88.7 & 91.2 & 1848 & 1.34 & 2.6 & 5905 & 1.10 & 1.16 & 1.37 & 1.39 & 2012Mat, 2012Bru \\
\hline KIC 10963065 & 21.0 & 102.6 & 105.5 & 2151 & 1.41 & 2.9 & 6060 & 1.03 & 1.07 & 1.20 & 1.23 & 2012App, 2012Mat, 2012Bru \\
\hline HD 181420 & 21.0 & 74.9 & 77.1 & 1573 & 0.98 & 3.7 & 6580 & 1.58 & 1.65 & 1.69 & 1.75 & 2009Bar, 2012Oze \\
\hline HD 49933 & 21.0 & 85.8 & 88.3 & 1805 & 1.04 & 2.4 & 6780 & 1.30 & 1.52 & 1.41 & 1.55 & 2009Ben, 2012Ree \\
\hline KIC 6603624 & 21.1 & 109.8 & 112.9 & 2312 & 1.56 & 2.6 & 5625 & 1.01 & 0.90 & 1.15 & 1.11 & 2012Mat, 2012App, 2012Bru \\
\hline KIC 12009504 & 21.1 & 87.8 & 90.3 & 1855 & 1.32 & 2.4 & 6065 & 1.26 & 1.27 & 1.43 & 1.44 & 2012App, 2012Mat, 2012Bru \\
\hline KIC 6116048 & 21.2 & 100.2 & 103.1 & 2130 & 1.44 & 2.7 & 5990 & 1.12 & 1.12 & 1.26 & 1.26 & 2012App, 2012Mat, 2012Bru \\
\hline 16 Cyg A & 21.3 & 102.5 & 105.4 & 2180 & 1.46 & 2.9 & 5825 & 1.11 & 1.05 & 1.24 & 1.22 & 2012Met \\
\hline KIC 8394589 & 21.4 & 108.9 & 112.0 & 2336 & 1.37 & 3.0 & 6114 & & 1.09 & & 1.19 & 2012App, 2012Bru \\
\hline KIC 11253226 & 21.8 & 77.0 & 79.1 & 1678 & 0.96 & 2.3 & 6605 & 1.46 & 1.82 & 1.63 & 1.77 & 2012App, 2012Sil, 2012Bru \\
\hline$\mu$ Arae & 21.8 & 89.5 & 91.9 & 1950 & 1.46 & 2.4 & 5813 & 1.14 & 1.29 & 1.36 & 1.43 & 2005Bou, 2005Baz \\
\hline HD 52265 & 21.8 & 98.5 & 101.2 & 2150 & 1.38 & 1.8 & 6100 & 1.27 & 1.27 & 1.34 & 1.34 & 2011Bal, 2012Giz \\
\hline KIC 6106415 & 21.9 & 103.9 & 106.7 & 2274 & 1.38 & 3.0 & 5990 & 1.12 & 1.18 & 1.24 & 1.26 & 2012Mat, 2012Bru \\
\hline KIC 10454113 & 22.1 & 104.7 & 107.6 & 2313 & 1.27 & 2.8 & 6120 & 1.16 & 1.24 & 1.25 & 1.27 & 2012App, 2012Sil, 2012Bru \\
\hline KIC 8379927 & 22.2 & 120.0 & 123.3 & 2669 & 1.37 & 2.4 & 5990 & 1.09 & 1.07 & 1.11 & 1.11 & 2012App, 2012Mat \\
\hline KIC 9139151 & 22.3 & 116.9 & 120.1 & 2610 & 1.40 & 2.1 & 6125 & 1.22 & 1.15 & 1.18 & 1.15 & 2012App, 2012Sil, 2012Bru \\
\hline 16 Cyg B & 22.4 & 115.3 & 118.4 & 2578 & 1.48 & 2.8 & 5750 & 1.07 & 1.07 & 1.13 & 1.14 & 2012Met \\
\hline KIC 9025370 & 22.4 & 132.3 & 135.9 & 2964 & 1.48 & 1.8 & 5660 & & 0.91 & & 0.98 & 2012App \\
\hline KIC 3427720 & 22.4 & 119.3 & 122.5 & 2674 & 1.48 & 3.1 & 6040 & & 1.12 & & 1.13 & 2012App, 2012Bru \\
\hline KIC 5184732 & 22.5 & 95.3 & 97.9 & 2142 & 1.43 & 3.3 & 5840 & 1.25 & 1.34 & 1.36 & 1.39 & 2012Mat, 2012Bru \\
\hline Sun & 22.7 & 134.4 & 138.0 & 3050 & 1.51 & 2.0 & 5777 & 1.00 & 0.97 & 1.00 & 0.99 & Sun \\
\hline KIC 8379927 & 22.8 & 120.4 & 123.6 & 2743 & 1.29 & 2.2 & 5900 & 1.09 & 1.13 & 1.11 & 1.12 & 2012Mat, 2012App \\
\hline$\alpha$ Cen A & 22.8 & 105.7 & 108.5 & 2410 & 1.36 & 1.7 & 5790 & 1.10 & 1.25 & 1.23 & 1.27 & 2004Bed, 2002The \\
\hline KIC 8006161 & 23.2 & 148.5 & 152.3 & 3444 & 1.68 & 2.2 & 5390 & 1.00 & 0.84 & 0.93 & 0.89 & 2012Mat, 2012App, 2012Bru \\
\hline $18 \mathrm{Sco}$ & 23.2 & 133.5 & 136.9 & 3100 & 1.54 & 1.8 & 5813 & 1.02 & 1.06 & 1.01 & 1.03 & 2011Baz, 2012Baz \\
\hline KIC 10644253 & 23.3 & 123.0 & 126.2 & 2866 & 1.47 & 2.6 & 6030 & & 1.22 & & 1.14 & 2012App, 2012Bru \\
\hline KIC 11772920 & 23.4 & 155.3 & 159.3 & 3639 & 1.52 & 1.8 & 5420 & & 0.84 & & 0.86 & 2012App \\
\hline KIC 9955598 & 23.5 & 152.6 & 156.5 & 3579 & 1.58 & 2.1 & 5410 & & 0.85 & & 0.88 & 2012App, 2012Bru \\
\hline$\alpha$ Cen B & 25.3 & 161.4 & 165.3 & 4090 & 1.48 & 1.6 & 5260 & 0.91 & 0.98 & 0.86 & 0.88 & 2005Kje, 2002The \\
\hline
\end{tabular}

